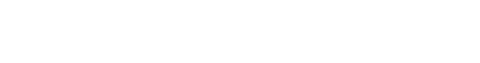

\section{A Case Report: Traditional Chinese Medicine Treatment in the Management of Exudative Infiltration Related to post-Cryoablation for Multiple Pulmonary Nodules}

Si Min Lee ${ }^{1}$, Xiaomei Zhang'2*, Mengqian Li ${ }^{1}$, Likun Che ${ }^{1}$, Rui Li ${ }^{1}$ and Xiaolin Yu ${ }^{1}$

${ }^{1}$ Post-graduate student, China

ISSN: $2637-773 \mathrm{X}$

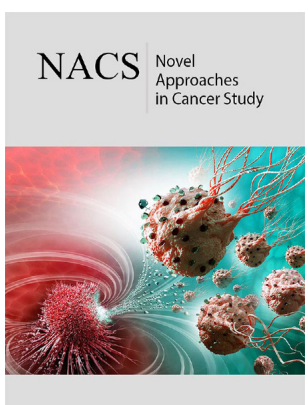

*Corresponding author: Xiaomei Zhang, Professor, Senior Consultant, Department of Respiratory Medicine, Beijing 100078, P.R. China

Submission: 地 February 01, 2021

Published: 留March 16, 2021

Volume 5 - Issue 5

How to cite this article: Si Min Lee, Xiaomei Zhang, Mengqian Li, Likun Che, Rui Li, Xiaolin Yu. A Case Report: Traditional Chinese Medicine Treatment in the Management of Exudative Infiltration Related to post-Cryoablation for Multiple Pulmonary Nodules. Nov Appro in Can Study. 5(5). NACS.000623. 2021. DOI: 10.31031/NACS.2021.05.000623

Copyright@Xiaomei Zhang, This article is distributed under the terms of the Creative Commons Attribution 4.0 International License, which permits unrestricted use and redistribution provided that the original author and source are credited.
${ }^{2}$ Senior Consultant, Department of Respiratory Medicine, China

\begin{abstract}
Percutaneous cryoablation is becoming a preferred option as one of the minimally invasive procedures in the management of tumor related cases due to its clearer visual field, promising anesthetic effect, perseverance of collagenous architecture as parts of its many advantages. In this case, a patient who was diagnosed with multiple pulmonary nodules with a high probability of malignancy, underwent this thermal ablative procedure. Lesions at the ablated zones viewed during the procedure are generally selflimiting, however, in our case ablated lesions which were marked by exudative infilitration and poor tissue healing persisted for over six months. The application of traditional Chinese medicine herbal treatment in this case was proven effective in managing such lesions, as well as its related signs and symptoms.
\end{abstract}

Keywords: Multiple pulmonary nodules; Ablation zone; Exudative infilitration; Tissue healing; Cryoablation; Traditional Chinese medicine

\section{Introduction}

A Pulmonary nodule is termed as "a rounded opacity, well or poorly defined, measuring up to $3 \mathrm{~cm}$ in diameter" by the Fleischner Society, in which nodules which are non-calcified are termed as potential malignant lesions [1,2]. An indeterminate solitary pulmonary nodule with high probability of malignancy is generally managed by a minimally invasive approach called video-assisted thoracoscopic surgery [3]. However, such procedure is less conducive in the case of multiple pulmonary nodules, especially when the nodules are scattered throughout multiple lobes of the lungs. Instead, cryoablation technique can be an alternative when managing cases which involve multiple lesions [4]. Lesions at the ablation zone which are commonly encountered prior to the procedure include cavitation, exudation, tissue damage, etc. are generally self-limiting under normal circumstances [5]. Reports show that enlargement of ablation zones are common, especially one month after the procedure, followed by gradual reduction in the size of the lesions, though scarring can be persistent [6]. On the contrary, cases of persisted cryoablation zones, as well as a typical shape transformation of the ablation zones are often associated with local recurrence [7].

Our team has treated over 2000 cases of pulmonary nodules with traditional Chinese herbal medicine. Based on our thorough clinical research, we attributed pulmonary nodules, especially nodules with a high probability of malignancy, as the result of intermingled phlegm, blood stasis and dampness are often due to deficiency of qi or stagnation of qi. Therefore, treatment principles of strengthening healthy qi, promoting the flow of qi, promoting the flow of blood and dissipating phlegm are applied when managing with pulmonary nodules $[8,9]$. In our humble opinion, ablated lesions prior to ablative techniques are also associated with local accumulation of phlegm, dampness, and blood stasis, in which efficient management is essential to promote the recovery of the patient, as well as to prevent a local recurrence. In this regard, we are presenting a case report which highlights the use of traditional Chinese medicine herbal treatment in managing exudative infiltration and tissue damage resulting from cryoablation treatment for multiple pulmonary nodules with a high risk of malignancy.

\section{Case Presentation}

A female patient, aged 56, discovered multiple bilateral nodules in her lungs in October 2019. The patient underwent a percutaneous cryoablation procedure in November 2019 at 
a local hospital in Guongdong city to manage three of her multiple nodules, which showed a high risk of malignancy according to the radiographic presentation, in which a nodule of $7 \mathrm{~mm}$ in diameter was located at the right upper lobe, another nodule of $7 \mathrm{~mm}$ in diameter was located in the left upper lobe, and another nodule of $6 \mathrm{~mm}$ in diameter was located at the left lower lobe. The patient then went for a routine CT follow-up in June 2020, and the radiographic imaging indicated ablated lesions marked by poor tissue healing with significant exudative infiltration at all three ablation zones with the presence of multiple ground-glass nodules and mixed ground-glass nodules as shown in Figure $1 \& 2$.

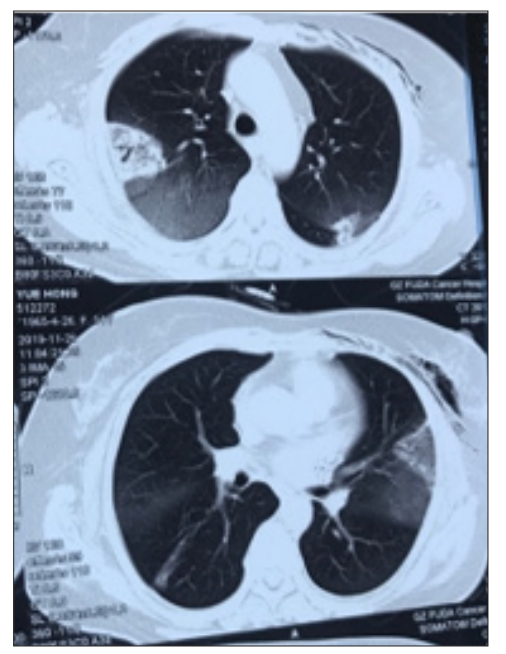

Figure 1: Ablated lesions was observed on the right upper lobe and left upper lobe of the lungs.

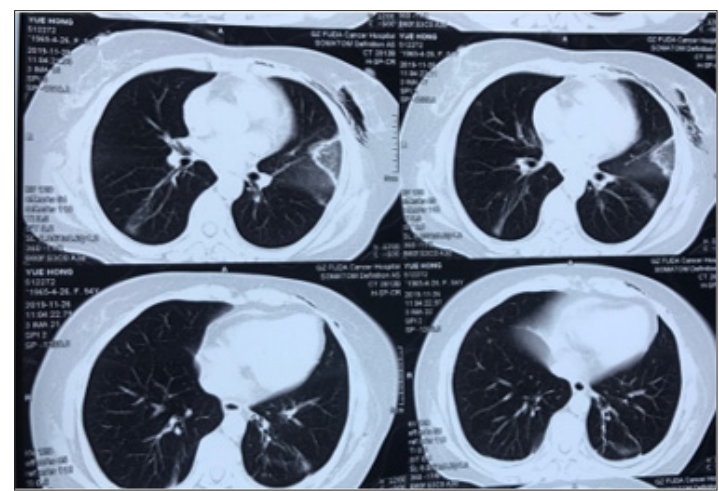

Figure 2: Ablated lesions was observed on the right upper lobe and left upper lobe of the lungs.

The patient first visited our outpatient clinic on July 2020 to seek medical consultation regarding her condition with the manifestation of shortness of breath, accompanied with chest oppression and chronic productive cough. Upon consultation, an herbal prescription, mainly comprised of Codonopsis Pilosula (Dangshen), Glehniae Radix (Beishashen), Ganoderma (Lingzhi), Sinapis Semen (Baijiezi), Pinellia Ternata (Banxia), Hedyotis Diffusa Willd (Baihuasheshecao), Viola Philippica (Zihuadiding), Agrimoniae Herba (Xianhecao), Solanum Nigrum (Longkui) and Astragali Radix (Huangqi) was prescribed to her for the duration of six months. The patient then went for a CT-screening on November 2020 , in which her CT images indicated no obvious ablated lesion with the absence of exudative infiltration at all three of her previous ablation zones. Tissue's damage resulted from the procedure was also fully recovered and no nodule was observed as shown in Figure 3 \& 4 . Her previous complaints of shortness of breath, chest oppression and chronic cough were significantly improved as well.

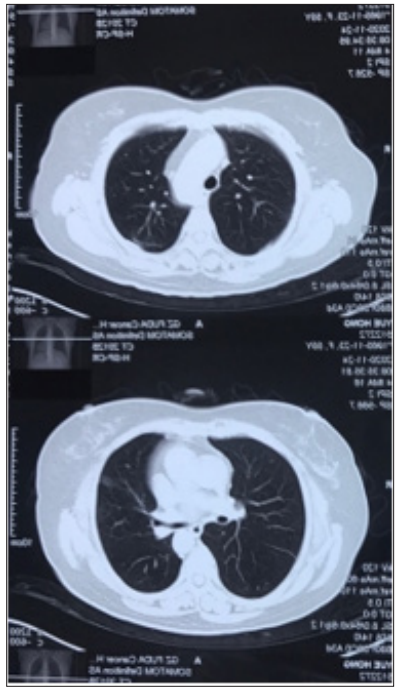

Figure 3: No ablated lesion was observed on the right upper lobe and left upper lobe of the lungs.

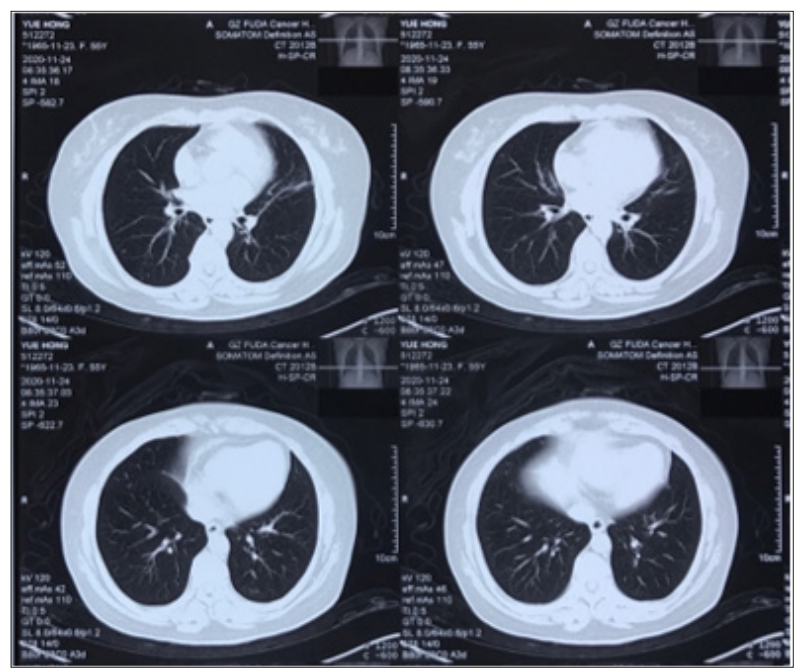

Figure 4: No ablated lesion was observed on the left lower lobe of the lungs.

\section{Discussion}

Our case highlighted the issues of exudative infiltration and tissue healing after the procedure of cryoablation. Generally, it will take about three to six months for the ablated area to shrink into a scar which is linear or nodular [10]. In our case, six months after the procedure, significant exudative infiltration and tissue damage were still observed radiologically, while the patient still manifested shortness of breath, accompanied with chest oppression and chronic cough, clinically. Some reports postulated that the ablation 
zone which did not reduce in size beyond six months is likely to be suggestive for recurrence of the tumour [10]. The prolonged recovery of the ablation zones in our case may also be procedural related, as cryoablation utilizes freezing-thawing technique in alternative cycles in order to destroy tumor affected tissues, but such method also causes direct injury to the neighboring cells and blood vessels; hence, an ablation zone which is too excessive can lead to unnecessary damages to the tissues and complications may arise as a result $[11,12]$. Apart from the risks for tumor recurrence, the ablation zone also increases the risks for local infection, resulting from tissue necrosis [13]. Although some reports suggest that the ablated lesions may disappear in 12 months after the procedure; however, given with the clinical manifestations experienced by our patient, as well as the risks possessed by prolonged ablation zones, the use of Chinese medicine herbal treatment can offer great help in resolving those issues.

\section{Conclusion}

Our case report suggested that apart from resolving the signs and symptoms experienced by our patient prior to a post percutaneous cryoablation treatment for MIA, herbal medicine can be effective in resolving exudative infilitration and potentially promote the healing of ablated lesions.

\section{Consent}

Written informed consent was obtained from patient for this case report.

\section{Acknowledgment}

We gratefully acknowledge our patient for allowing us to publish her case report.

\section{Authors' Contributions}

All authors have been involved in clinical diagnostic, evaluation and management in this case report. This manuscript was written and edited by both first author and corresponding author.

\section{Conflict of Interest}

The authors declare that they have no conflict of interests.

\section{Funding}

This case study was funded by Capital's Funds for Health Improvement and Research (CFH 2020-2-4202).

\section{References}

1. Hansell DM, Bankier AA, MacMahon H, Theresa C, Müller NL, et al (2008) Fleischner society: Glossary of terms for thoracic imaging. Radiol 246(3): 697-722.

2. Ost D, Fein A, Feinsilver SH (2003) The solitary pulmonary nodule. N Engl J Med 348(2): 2535-2543.

3. Davini F, Ricciardi S, Zirafa CC, Ilenia C, Gaetano R, et al. (2018) Treatment of pulmonary nodule: From VATS to RATS. J Vis Surg 4:36.

4. Bang HJ, Littrup PJ, Currier BP, Dylan JG, Hussein DA, et al. (2012) Percutaneous cryoablation of metastatic lesions from non-small-cell lung carcinoma: Initial survival, local control, and cost observations. J Vasc Inter Radiol 23(6): 761-769.

5. Sonntag PD, Hinshaw JL, Lubner MG, Christopher LB, Fred T Lee, et al. (2011) Thermal ablation of lung tumors. Surg Oncol Clin N Am 20(2): 369-387.

6. Ito N, Nakatsuka S, Inoue M, Hideki Yashiro, Sota Oguro, et al. (2012) Computed tomographic appearance of lung tumors treated with percutaneous cryoablation. J Vas Inter Radiol 23(8): 1043-1052.

7. Inoue M, Nakatsuka S, Jinzaki M (2014) Cryoablation of early-stage primary lung cancer. BioMed Research International, pp.1-8.

8. Man J, Zhang XM, Yu XL (2020) Syndrome differentiation and treatment of pulmonary nodules by Zhang Xiao-mei combined with CT images. CJTCMP 35(4): 1860-1862.

9. Chui JW, Liu YS, Zhang XM (2020) Treatment of pulmonary nodule from the perspectives of intermingling between phlegm and blood stasis. Global Traditional Chinese Medicine (Huangqiu Zhongyiyao) 13(10): 1737-1739.

10. Chan JWY, Lau RWH, Ng CSH (2020) Image-guided ablative therapies for lung tumors. Intech Open.

11. Lyons GR, Winokur RS, Pua BP (2019) Pulmonary cryoablation zones: More aggressive ablation is warranted in vivo. Am J Roentgenol 212(1): 195-200.

12. Chen CK, Chou HP, Sheu MH (2013) Image-guided lung tumor ablation: Principle, technique, and current status. J Chin Med Assoc 76(6): 303311.

13. Odisio BC, Richter M, Aloia TA, Claudius C, Kamran A, et al. (2016) Use of prophylactic antibiotics to prevent abscess formation following hepatic ablation in patients with prior enterobiliary manipulation. J Gastrointest Surg 20(8): 1428-1434. 